\title{
Snapshots of Modern Mathematics from Oberwolfach: Special Values of Zeta Functions and Areas of Triangles
}

\author{
Jürg Kramer and Anna-Maria von Pippich
}

\section{Some Recollections of the Rational Numbers The Rational Numbers and Their Countability}

A rational number is given as a fraction $\frac{a}{b}$, where $a, b$ belong to the set of integers $\mathbb{Z}$ and $b$ is nonzero. However, two rational numbers $\frac{a}{b}$ and $\frac{a^{\prime}}{b^{\prime}}$ are equal if the relation $a^{\prime} b=a b^{\prime}$ holds. This allows us to represent any rational number uniquely in its reduced form as $\frac{a}{b}$, where $a \in \mathbb{Z}$ and $b \in \mathbb{Z}_{>0}$ with greatest common divisor $(a, b)$ equal to 1 ; in other words, the set of rational numbers $\mathbb{Q}$ is given as

$$
\mathbb{Q}=\left\{\frac{a}{b} \mid(a, b) \in \mathbb{Z} \times \mathbb{Z}_{>0},(a, b)=1\right\} .
$$

The set of rational numbers is known to be countable, since we can enumerate the rational numbers in the well-known manner shown in Figure 1.

However, this counting process has the disadvantage that one rational number can occur several times. It is desirable to provide a counting procedure which lists each rational number exactly once. This will be done in the next subsection introducing the so-called Stern-Brocot tree, for which we refer to [7].

\section{The Stern-Brocot Tree}

The Stern-Brocot tree was discovered independently by the German number theorist Moritz Stern in 1858 and by the French clockmaker Achille Brocot in 1861.

Jürg Kramer is professor of mathematics at the Humboldt-Universität zu Berlin. He is supported by the DFG Graduate School "Berlin Mathematical School" and by the DFG International Research Training Group "Moduli and Automorphic Forms." His email address is kramer@math.hu-ber1 in.de.

Anna-Maria von Pippich is professor of mathematics at the Technische Universität Darmstadt. Her email address is pippich@ mathematik.tu-darmstadt. de.

For permission to reprint this article, please contact: reprint-permission@ams.org.

DOI: http://dx.doi.org/10.1090/noti1413

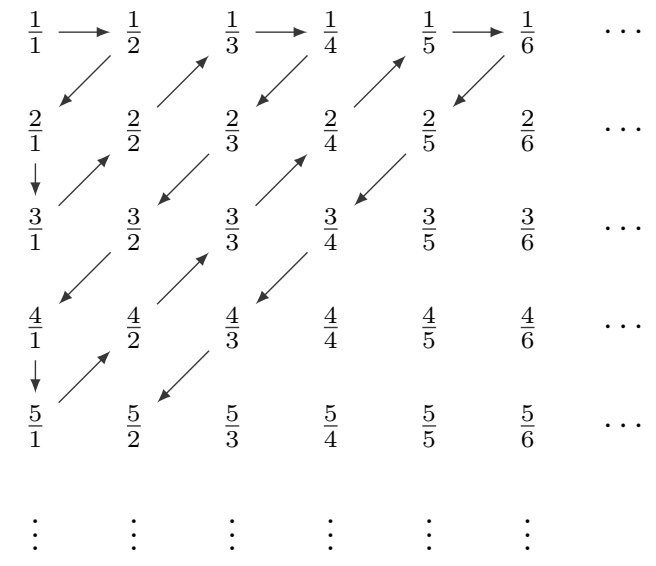

Figure 1 . The countability of $\mathbb{Q}$.

In order to construct the Stern-Brocot tree, we define the mediant of two rational numbers $\frac{a}{b}$ and $\frac{c}{d}$ by

$$
\frac{a}{b} \oplus \frac{c}{d}:=\frac{a+c}{b+d} .
$$

The vertices of the Stern-Brocot tree will be determined by positive rational numbers, starting with $1 / 1$ on the 0 -th level. A vertex of the tree that is labeled by the positive rational number $a / b$ gives rise to two new vertices by taking the mediant of $a / b$ with the nearest rational number to the left and to the right of $a / b$, respectively, already constructed in the tree (including $0 / 1$ and $1 / 0$, which do not belong to the tree). For example, the rational number $2 / 3$ gives rise to the two new vertices $3 / 5$ and $3 / 4$, since $3 / 5$ is the mediant of $2 / 3$ and $1 / 2$, which is nearest to $2 / 3$ to the left, while $3 / 4$ is the mediant of $2 / 3$ and $1 / 1$, which is nearest to $2 / 3$ to the right. The resulting binary tree is depicted in Figure 2.

As indicated in Figure 2, it is convenient to keep track of all the rational numbers constructed in this way by successively copying them to the next level of the construction. On each level (starting with the 0-th level) 


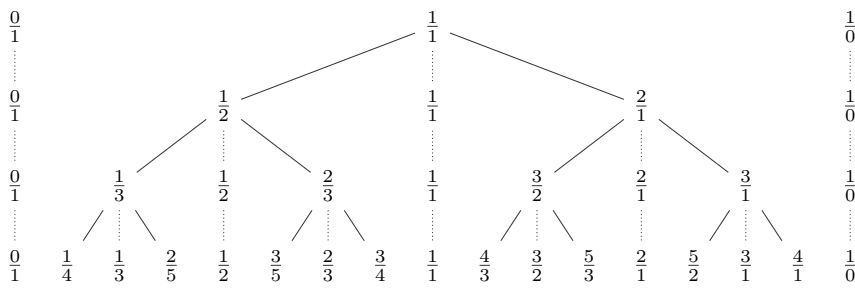

Figure 2. The Stern-Brocot tree.

we thus collect the rational numbers constructed so far in a strictly ordered manner from left to right, starting with $0 / 1$ and ending with $1 / 0$ (which is not a rational number, but can be thought of as a quantity larger than any positive rational number). In the sequel we will call two rational numbers neighboring fractions if they are next to each other on the same level in the tree.

\section{Properties of the Stern-Brocot Tree}

We now list and prove some of the main properties of the Stern-Brocot tree.

(1) We claim that if $\frac{a}{b}<\frac{c}{d}$ are two neighboring fractions on the same level of the Stern-Brocot tree, then the following inequalities involving the mediant hold on the next level:

$$
\frac{a}{b}<\frac{a}{b} \oplus \frac{c}{d}<\frac{c}{d} .
$$

In order to prove these inequalities, we interpret the fractions $\frac{a}{b}$ and $\frac{c}{d}$ as slopes of straight lines through the origin of the $x, y$-plane. It is then immediately checked that the straight line through the origin with slope $\frac{a}{b} \oplus \frac{c}{d}$ lies between the lines with slopes $\frac{a}{b}$ and $\frac{c}{d}$, respectively. (We recommend you make a sketch to convince yourself of this!)

(2) We claim that if $\frac{a}{b}<\frac{c}{d}$ are two neighboring fractions on the same level of the Stern-Brocot tree, then the equation $b c-a d=1$ holds. This is easily seen by induction on the level: On the 0 -th level, the claim is obviously true. Let us thus assume that the claim holds on the $n$-th level for the neighboring fractions $\frac{a}{b}<\frac{c}{d}$. Passing to the $(n+1)$-st level the proof of the previous claim leads to the neighboring fractions

$$
\frac{a}{b}<\frac{a+c}{b+d}<\frac{c}{d},
$$

for which we compute (using the induction hypothesis)

$$
\begin{aligned}
& b(a+c)-a(b+d)=b c-a d=1, \\
& (b+d) c-(a+c) d=b c-a d=1 .
\end{aligned}
$$

This completes the induction step.

(3) By what we have just shown, we find that the fractions occurring in the Stern-Brocot tree are always positive rational numbers in reduced form. It is not hard to see that every positive rational number occurs in the Stern-Brocot tree. Thus, we have established a bijection between the positive rational numbers and the vertices of the Stern-Brocot tree.

\section{Special Values of the Riemann Zeta Function} The Computation of $\zeta(2)$

The Riemann zeta function is defined as the series

$$
\zeta(s):=\sum_{n=1}^{\infty} \frac{1}{n^{s}},
$$

where $s \in \mathbb{C}$ with $\operatorname{Re}(s)>1$. In this subsection our aim is to compute the special value $\zeta(2)$. We will complete this task by relating $\zeta(2)$ to the area of two triangles; we will follow the article [8].

To do this, we start by rewriting the term $1 / n^{2}\left(n \in \mathbb{Z}_{>0}\right)$ in the form

$$
\frac{1}{n^{2}}=-\left.\frac{1}{n} \cdot \frac{e^{-n x}}{n}\right|_{0} ^{\infty}=\int_{0}^{\infty} \frac{e^{-n x}}{n} \mathrm{~d} x .
$$

Thus, the term $1 / n^{2}$ equals the area of the region to the right of the $y$-axis between the exponential curve defined by the equation $y=e^{-n x} / n$ and the $x$-axis; see Figure 3 .

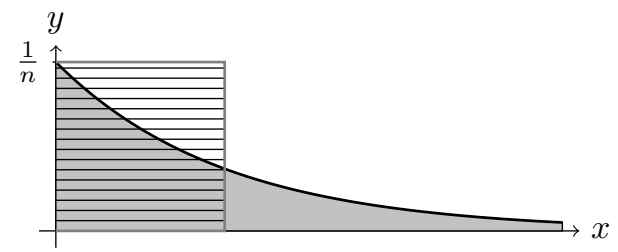

Figure $3.1 / n^{2}$ as the area below an exponential curve.

Therefore, the value of the series

$$
\zeta(2)=1+\frac{1}{2^{2}}+\frac{1}{3^{2}}+\cdots+\frac{1}{n^{2}}+\cdots
$$

can geometrically be visualized as depicted in Figure 4 .

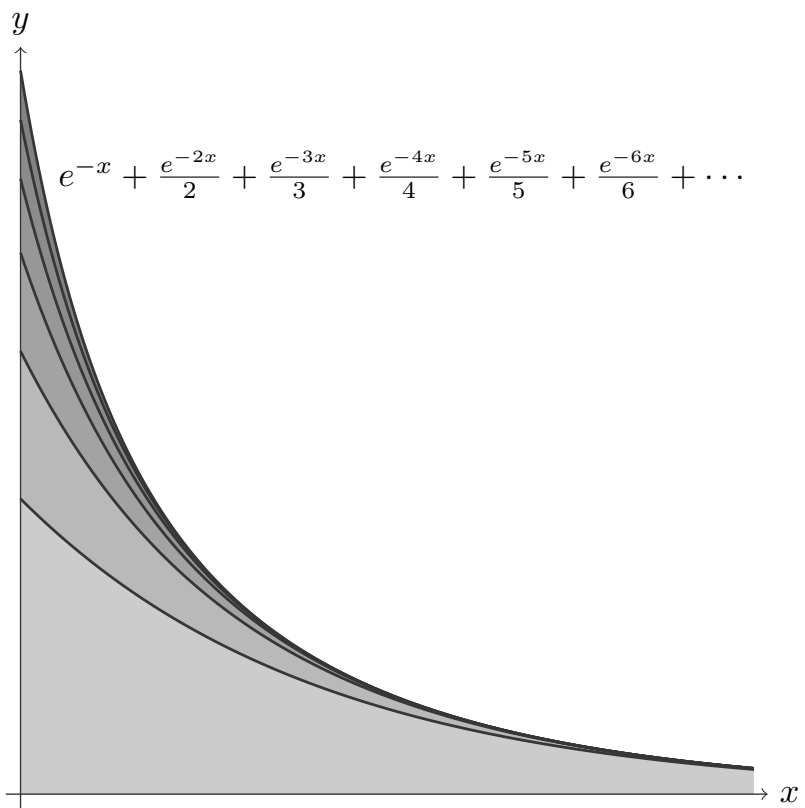

Figure 4. $\zeta(2)$ as a sum of areas of curved triangles. 
Using the well-known Taylor expansion for the logarithm function

$$
\sum_{n=1}^{\infty} \frac{e^{-n x}}{n}=-\log \left(1-e^{-x}\right)
$$

in conjunction with relation (1), we obtain

$$
\begin{aligned}
\zeta(2) & =\sum_{n=1}^{\infty} \frac{1}{n^{2}}=\sum_{n=1}^{\infty} \int_{0}^{\infty} \frac{e^{-n x}}{n} \mathrm{~d} x=\int_{0}^{\infty} \sum_{n=1}^{\infty} \frac{e^{-n x}}{n} \mathrm{~d} x \\
& =-\int_{0}^{\infty} \log \left(1-e^{-x}\right) \mathrm{d} x .
\end{aligned}
$$

This proves that $\zeta(2)$ equals the area of the region $A$ determined by the curve $C$ defined by the equation

$$
C: e^{-y}+e^{-x}=1
$$

in the $x, y$-plane for $0 \leq x<\infty$; see Figure 5 . In order to

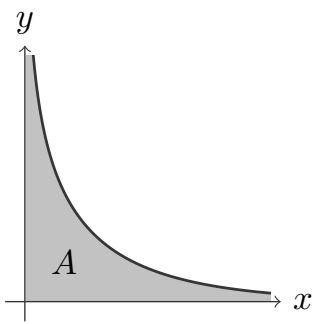

Figure 5. $\zeta(2)$ as area of the region $A$.

compute the area of $A$, we make the change of variables

$$
\begin{aligned}
(\alpha, \beta) & \mapsto(x, y)=\varphi(\alpha, \beta) \\
& :=\left(\log \left(\frac{\sin (\alpha+\beta)}{\sin (\alpha)}\right), \log \left(\frac{\sin (\alpha+\beta)}{\sin (\beta)}\right)\right)
\end{aligned}
$$

from the $\alpha, \beta$-plane to the $x, y$-plane. By the integral transformation formula (a two-dimensional generalization of integration by substitution), we then derive

$$
\operatorname{area}(A)=\iint_{A} \mathrm{~d} x \mathrm{~d} y=\iint_{B}\left|\operatorname{det}\left(\frac{\partial \varphi}{\partial \alpha}, \frac{\partial \varphi}{\partial \beta}\right)\right| \mathrm{d} \alpha \mathrm{d} \beta,
$$

where $B$ denotes the region in the $\alpha, \beta$-plane which is mapped bijectively to the region $A$ in the $x, y$-plane by the map $\varphi$. A straightforward calculation shows that the determinant of the Jacobian of $\varphi$ is given by

$$
\begin{aligned}
& \operatorname{det}\left(\frac{\partial \varphi}{\partial \alpha}, \frac{\partial \varphi}{\partial \beta}\right) \\
& \quad=\left|\begin{array}{cc}
\cot (\alpha+\beta)-\cot (\alpha) & \cot (\alpha+\beta) \\
\cot (\alpha+\beta) & \cot (\alpha+\beta)-\cot (\beta)
\end{array}\right|=1,
\end{aligned}
$$

using the addition formula

$$
\cot (\alpha+\beta)=(\cot (\alpha) \cot (\beta)-1) /(\cot (\alpha)+\cot (\beta))
$$

for the cotangent. Furthermore, it can be shown that the region $B$ is given as the union of the following two triangles:

$$
\begin{aligned}
& B_{1}:=\left\{(\alpha, \beta) \mid 0 \leq \alpha \leq \frac{\pi}{3} ; \alpha \leq \beta \leq \frac{\pi}{2}-\frac{\alpha}{2}\right\}, \\
& B_{2}:=\left\{(\alpha, \beta) \mid 0 \leq \beta \leq \frac{\pi}{3} ; \beta \leq \alpha \leq \frac{\pi}{2}-\frac{\beta}{2}\right\},
\end{aligned}
$$

as depicted in Figure 6. Using
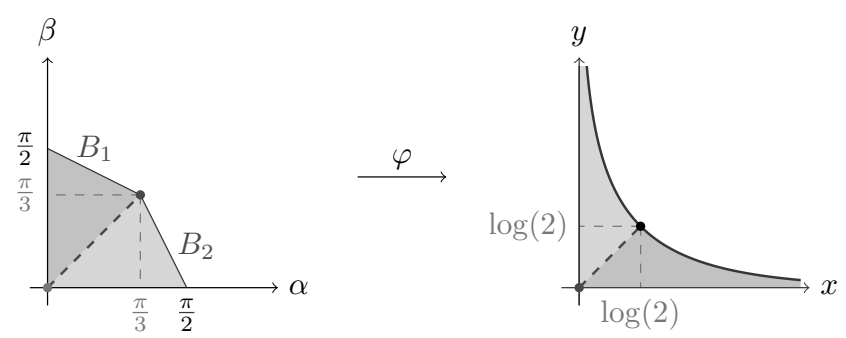

Figure 6. $\zeta(2)$ as area of the two triangles $B_{1}$ and $B_{2}$.

$$
\operatorname{area}\left(B_{1}\right)=\frac{1}{2} \cdot \frac{\pi}{2} \cdot \frac{\pi}{3}=\operatorname{area}\left(B_{2}\right),
$$

we obtain

$$
\zeta(2)=\operatorname{area}(A)=\operatorname{area}(B)=\operatorname{area}\left(B_{1}\right)+\operatorname{area}\left(B_{2}\right)=\frac{\pi^{2}}{6},
$$

which is the well-known value already determined by the Swiss mathematician Leonhard Euler (1707-1783) in his solution to the so-called "Basel Problem."

\section{Interpreting $\zeta(2)$ as Volume of a Moduli Space}

The special value $\zeta$ (2) of the Riemann zeta function has another geometric interpretation; namely, it also gives the volume of the moduli space $\mathscr{A}_{1}$ of elliptic curves, that is, of the moduli space of smooth projective curves of genus $g=1$. More specifically, this means that the points of $\mathscr{A}_{1}$ are in bijection with the isomorphism classes of elliptic curves over $\mathbb{C}$, the bijection being given by assigning to the point $[z] \in \mathscr{A}_{1}$ the isomorphism class of the elliptic curve $\mathbb{C} /(\mathbb{Z} \oplus \mathbb{Z} Z)$. Therefore, $\mathscr{A}_{1}$ can be realized as the quotient space $\mathrm{SL}_{2}(\mathbb{Z}) \backslash \mathbb{U}$, where $\mathrm{SL}_{2}(\mathbb{Z})$ is the modular group acting by fractional linear transformations on the hyperbolic upper half-plane $\mathbb{U}:=\{z=x+i y \in \mathbb{C} \mid y>0\}$. It is known that the quotient space $\mathrm{SL}_{2}(\mathbb{Z}) \backslash \mathbb{W}$ is in continuous bijection with the fundamental domain

$$
\mathscr{F}:=\left\{z=x+i y \in \mathbb{Q} \mid-1 / 2<x \leq 1 / 2, x^{2}+y^{2} \geq 1\right\}
$$

depicted in Figure 7.

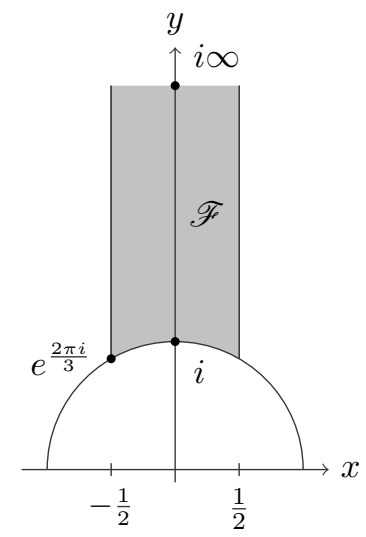

Figure 7. Fundamental domain $\mathscr{F}$ for $\mathrm{SL}_{2}(\mathbb{Z})$ acting on आ.

By means of these identifications, the volume of $\mathscr{A}_{1}$ with respect to the normalized hyperbolic metric $\mathrm{d} \mu_{1}$ can 
be computed as

$$
\begin{aligned}
& =\int_{\mathscr{A}_{1}} \mathrm{dol}\left(\mathscr{A}_{1}\right) \\
& =\frac{1}{4 \pi} \int_{-1 / 2}^{+1 / 2} \int_{\sqrt{1-x^{2}}}^{\infty} \frac{\mathrm{d} x \wedge \mathrm{d} y}{y^{2}}=\frac{1}{4 \pi} \int_{-1 / 2}^{+1 / 2} \frac{1}{\sqrt{1-x^{2}}} \mathrm{~d} x
\end{aligned}
$$

recalling the identity $\zeta(2)=\pi^{2} / 6$ from the subsection "The Computation of $\zeta(2)$." Using the functional equation of the Riemann zeta function

$$
\pi^{-s / 2} \Gamma\left(\frac{s}{2}\right) \zeta(s)=\pi^{-(1-s) / 2} \Gamma\left(\frac{1-s}{2}\right) \zeta(1-s),
$$

the formula for the volume of $\mathscr{A}_{1}$ can be rewritten in the simplified form

$$
\operatorname{vol}\left(\mathscr{A}_{1}\right)=-\zeta(-1)
$$

\section{Special Values of the Mordell-Tornheim Zeta Function}

\section{The Setup}

The Mordell-Tornheim zeta function is defined as the double series

$$
\zeta_{\mathrm{MT}}\left(s_{1}, s_{2} ; s_{3}\right):=\sum_{m, n=1}^{\infty} \frac{1}{m^{s_{1}} n^{s_{2}}(m+n)^{s_{3}}},
$$

where $s_{1}, s_{2}, s_{3} \in \mathbb{C}$ with $\operatorname{Re}\left(s_{1}\right) \geq \operatorname{Re}\left(s_{2}\right) \geq \operatorname{Re}\left(s_{3}\right)>1$ (see [5]). In this section our aim is to compute the special value

$$
\zeta_{\mathrm{MT}}(2,2 ; 2)=\sum_{m, n=1}^{\infty} \frac{1}{m^{2} n^{2}(m+n)^{2}} .
$$

We will be able to do this by relating the special value $\zeta_{\mathrm{MT}}(2,2 ; 2)$ to the area of the region $F$ determined by the curve $C$ defined by the equation

$$
C: \sqrt{y}+\sqrt{x}=1
$$

in the $x, y$-plane for $0 \leq x \leq 1$; see Figure 8 . Using

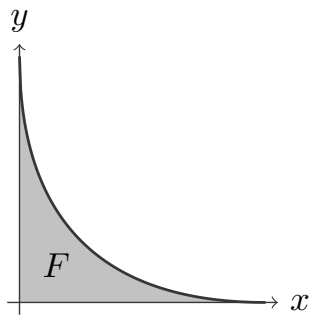

Figure 8 . The area of the region $F$.

integration it is easy to compute the area of $F$ simply as

$$
\begin{aligned}
\operatorname{area}(F) & =\int_{0}^{1}(1-\sqrt{x})^{2} \mathrm{~d} x=\int_{0}^{1}(1-2 \sqrt{x}+x) \mathrm{d} x \\
& =\left.\left(x-\frac{4}{3} x^{3 / 2}+\frac{1}{2} x^{2}\right)\right|_{0} ^{1}=\frac{1}{6} .
\end{aligned}
$$

The Computation of $\zeta_{\mathrm{MT}}(2,2 ; 2)$

In order to compute $\zeta_{\mathrm{MT}}(2,2 ; 2)$, we will interpret it as the area of the region $F$. For this purpose we will approximate the region $F$ by triangles $\Delta_{m, n}$ parametrized by positive coprime integers $m, n$ determined by the Stern-Brocot tree. More specifically, we consider on each level (starting with the 0-th level) all neighboring fractions $\frac{a}{b}<\frac{c}{d}$ in the Stern-Brocot tree. For convenience in the subsequent calculations, we rewrite these fractions in the form

$$
\frac{a}{b}=\frac{m^{\prime}}{m-m^{\prime}} \quad \text { and } \quad \frac{c}{d}=\frac{n^{\prime}}{n-n^{\prime}}
$$

by setting $m^{\prime}:=a, m:=a+b$ and $n^{\prime}:=c, n:=c+d$. Since $\frac{a}{b}<\frac{c}{d}$ are neighboring fractions in the Stern-Brocot tree, we have the relation

$$
m^{\prime} n-m n^{\prime}=a(c+d)-(a+b) c=-1,
$$

using observation (2) from the subsection "Properties of the Stern-Brocot Tree." In order to obtain the desired triangles $\Delta_{m, n}$, we need the successor of (3) in the SternBrocot tree on the next level, which is given as the mediant

$$
\frac{m^{\prime}+n^{\prime}}{\left(m-m^{\prime}\right)+\left(n-n^{\prime}\right)}=\frac{m^{\prime}+n^{\prime}}{(m+n)-\left(m^{\prime}+n^{\prime}\right)} .
$$

We next consider the three lines whose slopes are determined by the negative reciprocals of the fractions (3), (5) and which are tangent to the curve $C$ in the points

$$
\begin{aligned}
& T_{1}:=\left(\frac{m^{\prime 2}}{m^{2}}, \frac{\left(m-m^{\prime}\right)^{2}}{m^{2}}\right), \\
& T_{2}:=\left(\frac{n^{\prime 2}}{n^{2}}, \frac{\left(n-n^{\prime}\right)^{2}}{n^{2}}\right), \\
& T_{3}:=\left(\frac{\left(m^{\prime}+n^{\prime}\right)^{2}}{(m+n)^{2}}, \frac{\left((m+n)-\left(m^{\prime}+n^{\prime}\right)\right)^{2}}{(m+n)^{2}}\right),
\end{aligned}
$$

respectively. These three lines are explicitly given by the formulae

$t_{1}(x):=-\frac{m-m^{\prime}}{m^{\prime}} x+\frac{m-m^{\prime}}{m}$,

$t_{2}(x):=-\frac{n-n^{\prime}}{n^{\prime}} x+\frac{n-n^{\prime}}{n}$,

$t_{3}(x):=-\frac{(m+n)-\left(m^{\prime}+n^{\prime}\right)}{m^{\prime}+n^{\prime}} x+\frac{(m+n)-\left(m^{\prime}+n^{\prime}\right)}{m+n}$,

respectively. The triangle $\Delta_{m, n}$ is now determined by the three intersection points $P_{1}, P_{2}, P_{3}$ of the lines $t_{1}(x)$ with $t_{2}(x), t_{1}(x)$ with $t_{3}(x)$, and $t_{2}(x)$ with $t_{3}(x)$, respectively; see Figure 9. A straightforward calculation yields

$$
\begin{aligned}
P_{1} & =\left(x_{1}, y_{1}\right)=\left(\frac{m^{\prime} n^{\prime}}{m n}, \frac{\left(m-m^{\prime}\right)\left(n-n^{\prime}\right)}{m n}\right), \\
P_{2} & =\left(x_{2}, y_{2}\right) \\
& =\left(\frac{m^{\prime}\left(m^{\prime}+n^{\prime}\right)}{m(m+n)}, \frac{\left(m-m^{\prime}\right)\left((m+n)-\left(m^{\prime}+n^{\prime}\right)\right)}{m(m+n)}\right), \\
P_{3} & =\left(x_{3}, y_{3}\right) \\
& =\left(\frac{n^{\prime}\left(m^{\prime}+n^{\prime}\right)}{n(m+n)}, \frac{\left(n-n^{\prime}\right)\left((m+n)-\left(m^{\prime}+n^{\prime}\right)\right)}{n(m+n)}\right) .
\end{aligned}
$$

Using $N:=m n(m+n)$, the area of the triangle $\Delta_{m, n}$ is 


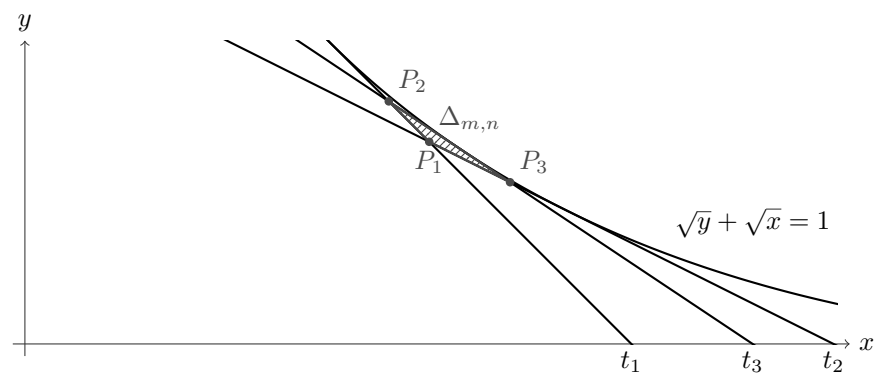

Figure 9. The triangle $\Delta_{m, n}$.

then given by the formula

$$
\begin{aligned}
& \operatorname{area}\left(\Delta_{m, n}\right) \\
& \quad=\frac{1}{2}\left|\operatorname{det}\left(\begin{array}{ll}
x_{2}-x_{1} & x_{3}-x_{1} \\
y_{2}-y_{1} & y_{3}-y_{1}
\end{array}\right)\right|=\frac{1}{2 N^{2}}\left|\operatorname{det}\left(\begin{array}{ll}
A & B \\
C & D
\end{array}\right)\right|,
\end{aligned}
$$

where

$$
\begin{aligned}
& A:=m^{\prime} n\left(m^{\prime}+n^{\prime}\right)-m^{\prime} n^{\prime}(m+n), \\
& B:=m n^{\prime}\left(m^{\prime}+n^{\prime}\right)-m^{\prime} n^{\prime}(m+n), \\
& C:=\left(m-m^{\prime}\right)\left[n(m+n)-n\left(m^{\prime}+n^{\prime}\right)-(m+n)\left(n-n^{\prime}\right)\right], \\
& D:=\left(n-n^{\prime}\right)\left[m(m+n)-m\left(m^{\prime}+n^{\prime}\right)-(m+n)\left(m-m^{\prime}\right)\right] .
\end{aligned}
$$

Using (4), a direct calculation now gives

$$
\begin{aligned}
& \operatorname{area}\left(\Delta_{m, n}\right) \\
& =\frac{1}{2 N^{2}}\left|\operatorname{det}\left(\begin{array}{cc}
m^{\prime}\left(m^{\prime} n-m n^{\prime}\right) & n^{\prime}\left(m n^{\prime}-m^{\prime} n\right) \\
\left(m-m^{\prime}\right)\left(m n^{\prime}-m^{\prime} n\right) & \left(n-n^{\prime}\right)\left(m^{\prime} n-m n^{\prime}\right)
\end{array}\right)\right| \\
& =\frac{1}{2 N^{2}}\left|\operatorname{det}\left(\begin{array}{cc}
-m^{\prime} & n^{\prime} \\
\left(m-m^{\prime}\right) & -\left(n-n^{\prime}\right)
\end{array}\right)\right| \\
& =\frac{1}{2 N^{2}}\left|m^{\prime}\left(n-n^{\prime}\right)-n^{\prime}\left(m-m^{\prime}\right)\right| \\
& =\frac{1}{2 N^{2}}\left|m^{\prime} n-m n^{\prime}\right| .
\end{aligned}
$$

Using (4) once again, we find that the area of the triangle $\Delta_{m, n}$ is given by the formula

$$
\operatorname{area}\left(\Delta_{m, n}\right)=\frac{1}{2} \frac{1}{m^{2} n^{2}(m+n)^{2}} .
$$

Note that the triangles $\Delta_{m, n}$ exhaust the area under the curve $C$ (for $0 \leq x \leq 1$ ) as $m, n$ range through all positive coprime integers; see Figure 10. (Why?) We thus obtain

$$
\begin{aligned}
\operatorname{area}(F) & =\sum_{\substack{m, n=1 \\
(m, n)=1}}^{\infty} \operatorname{area}\left(\Delta_{m, n}\right) \\
& \Longleftrightarrow \frac{1}{2} \sum_{\substack{m, n=1 \\
(m, n)=1}}^{\infty} \frac{1}{m^{2} n^{2}(m+n)^{2}}=\frac{1}{6} .
\end{aligned}
$$

Finally, we end up with

$$
\zeta_{\mathrm{MT}}(2,2 ; 2)=\zeta(6) \sum_{\substack{m, n=1 \\(m, n)=1}}^{\infty} \frac{1}{m^{2} n^{2}(m+n)^{2}}=\frac{\pi^{6}}{945} \cdot \frac{1}{3}=\frac{\pi^{6}}{2835},
$$

using the identity $\zeta(6)=8 \zeta(2)^{3} / 35=\pi^{6} / 945$, which is derived from well-known relations between values of the

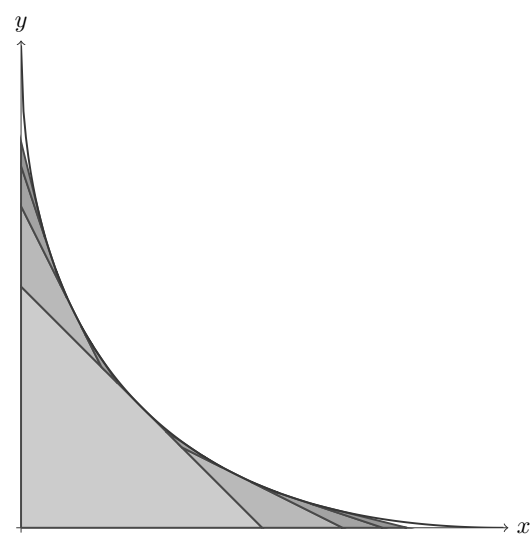

Figure 10. $\zeta_{\mathrm{MT}}(2,2 ; 2)$ as a sum of the triangles $\Delta_{m, n}$.

Riemann zeta function at the even positive integers (see for example [10]).

\section{Relation to Current Research}

In generalization of the one-dimensional situation discussed in the subsection "Interpreting $\zeta(2)$ as Volume of a Moduli Space," special values of the Riemann zeta function at even positive integers arise in the computation of the volume of the moduli space $\mathscr{A}_{g}$ of $g$-dimensional principally polarized Abelian varieties. Using the functional equation, these volume formulas can be rewritten in terms of special values of the Riemann zeta function at negative odd integers. More specifically, one can realize the moduli spaces $\mathscr{A}_{q}$ as the quotient space $\mathrm{Sp}_{g}(\mathbb{Z}) \backslash \mathbb{H}_{q}$, where $\mathrm{Sp}_{g}(\mathbb{Z})$ is the Siegel modular group acting on the hyperbolic Siegel upper half-space $\mathbb{t}_{g}$ of degree $g$. Using a suitably normalized volume element $\mathrm{d} \mu_{g}$, the generalization of formula (2) is given as

$$
\operatorname{vol}\left(\mathscr{A}_{g}\right)=\int_{\mathscr{A}_{g}} \mathrm{~d} \mu_{g}=2 \prod_{j=1}^{g} \frac{(-1)^{j} j !}{(2 j) !} \zeta(1-2 j)
$$

(see [9]). From a conceptual point of view, the formula for the volume of $\mathscr{A}_{g}$ has the following two interpretations: The algebraic geometric interpretation of $\operatorname{vol}\left(\mathscr{A}_{g}\right)$ is that of an intersection product of line bundles (or, equivalently, of divisors). Namely, $\operatorname{vol}\left(\mathscr{A}_{g}\right)$ equals the $\operatorname{dim}\left(\mathscr{A}_{g}\right)$-fold selfintersection number of the Hodge bundle $\omega_{g}$ of $\mathscr{A}_{g}$. On the other hand, the differential geometric interpretation is given as

$$
\operatorname{vol}\left(\mathscr{A}_{g}\right)=\int_{\mathscr{A}_{g}} \underbrace{\mathrm{c}_{1}\left(\omega_{g},\|\cdot\|\right) \wedge \cdots \wedge \mathrm{c}_{1}\left(\omega_{g},\|\cdot\|\right)}_{\operatorname{dim}\left(\mathscr{A}_{g}\right) \text {-times }},
$$

where $c_{1}\left(\omega_{g},\|\cdot\|\right)$ denotes the first Chern form of the Hodge bundle $\omega_{g}$, equipped with the Petersson metric $\|\cdot\|$. The fact that these two interpretations lead to the same result is a consequence of Chern-Weil theory, which assumes that the metric in question is smooth (see for example [3]). In the present case the metric $\|\cdot\|$ under consideration becomes logarithmically singular when one approaches the boundary of $\mathscr{A}_{g}$; nevertheless, Chern-Weil 
theory continues to hold true thanks to a deep result of Mumford (see [6]).

After this preliminary discussion, we are now able to provide a geometric interpretation of the special value $\zeta_{\mathrm{MT}}(2,2 ; 2)$ of the Mordell-Tornheim zeta function. Associated to the moduli space $\mathscr{A}_{1}$ of elliptic curves, there is the universal elliptic curve $\mathscr{B}_{1}$, which is a fibration $\pi_{1}: \mathscr{B}_{1} \rightarrow \mathscr{A}_{1}$ so that the fiber of $\pi_{1}$ over $[z] \in \mathscr{A}_{1}$ is the elliptic curve $\mathbb{C} /(\mathbb{Z} \oplus \mathbb{Z} z)$. More specifically, the universal elliptic curve is given as

$$
\mathscr{B}_{1}=\mathrm{SL}_{2}(\mathbb{Z}) \ltimes \mathbb{Z}^{2} \backslash \mathbb{U} \times \mathbb{C} .
$$

In analogy to the Hodge bundle $\omega_{1}$ on $\mathscr{A}_{1}$, there is a distinguished line bundle $\mathscr{L}_{1}$ on $\mathscr{B}_{1}$ which carries a natural metric $\|\cdot\|_{\Theta}$. As before, we can now compare the 2 -fold self-intersection number $\mathscr{L}_{1} \cdot \mathscr{L}_{1}$ (note that $\operatorname{dim}\left(\mathscr{B}_{1}\right)=2$ ) of the line bundle $\mathscr{L}_{1}$ with the quantity

$$
\operatorname{vol}\left(\mathscr{B}_{1}\right)=\int_{\mathscr{B}_{1}} \mathrm{c}_{1}\left(\mathscr{L}_{1},\|\cdot\|_{\Theta}\right) \wedge \mathrm{c}_{1}\left(\mathscr{L}_{1},\|\cdot\|_{\Theta}\right) .
$$

Since the singularities of the metric $\|\cdot\|_{\Theta}$ as one approaches the boundary of $\mathscr{B}_{1}$ are worse than logarithmic, it turns out that the two quantities under consideration are no longer equal. It turns out that the difference is measured by the special value $\zeta_{\mathrm{MT}}(2,2 ; 2)$; that is, we have

(6)

$$
\frac{\zeta_{\mathrm{MT}}(2,2 ; 2)}{\zeta(6)}=\mathscr{L}_{1} \cdot \mathscr{L}_{1}-\int_{\mathscr{B}_{1}} \mathrm{c}_{1}\left(\mathscr{L}_{1},\|\cdot\|_{\Theta}\right) \wedge \mathrm{c}_{1}\left(\mathscr{L}_{1},\|\cdot\|_{\Theta}\right) .
$$

This result can be explained as follows: The compactification $\overline{\mathscr{B}}_{1}$ of $\mathscr{B}_{1}$ over the one-point compactification $\overline{\mathscr{A}}_{1}$ of

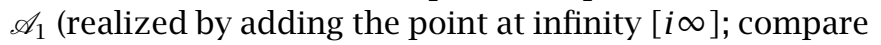
with the fundamental domain $\mathscr{F}$ ) is obtained by adding a curve of genus 0 with a node over $[i \infty]$. By blowing up the node and subsequently blowing up the arising new nodes, one obtains an infinite chain of blow-ups:

$$
\ldots \rightarrow \overline{\mathscr{B}}_{1}^{\prime \prime} \longrightarrow \overline{\mathscr{B}}_{1}^{\prime} \longrightarrow \overline{\mathscr{B}}_{1} \text {. }
$$

By taking the pullbacks of the line bundle $\mathscr{L}_{1}$ onto the various blowups, it turns out that on each level the difference between the respective self-intersection numbers and the integrals over the wedge product of the respective Chern forms decreases by the summands defining the series $\zeta_{\mathrm{MT}}(2,2 ; 2)$. In the limiting case, ChernWeil theory holds, which then provides the claimed value for the difference (6). For more details, we refer to [4] and [2].

The next steps in research consist in generalizing the results obtained for $g=1$ to the case of the universal Abelian variety $\pi_{g}: \mathscr{B}_{g} \rightarrow \mathscr{A}_{g}$ for $g>1$ and more general mixed Shimura varieties, as well as in translating the geometric results thus obtained to the Arakelovian setting based on the results established in [1].

\section{References}

1. J. I. Burgos GiL, J. KrAmer, and U. KüHN, Arithmetic characteristic classes of automorphic vector bundles, Doc. Math. 10 (2005), 619-716 (electronic). MR 2218402
2. cobi line bundle, in Recent Advances in Hodge Theory: Period Domains, Algebraic Cycles, and Arithmetic, M. Kerr, G. Pearlstein (eds.), pp. 45-77, London Mathematical Society Lecture Notes Series 427, Cambridge University Press, Cambridge, UK, arxiv:1405.3075v1 2014.

3. P. GRIFFITHS and J. HARRIS, Principles of Algebraic Geometry, Wiley-Interscience, New York, 1978. MR 0507725

4. J. KRAmER, A Geometrical Approach to Jacobi Forms, Revisited, 2014, Oberwolfach reports from the workshop on modular forms held April 22-May 2, 2014, organized by J. H. Bruinier, A. Ichino, T. Ikeda and Ö. Imamoglu, Report No. 22/2014. DOI: 10.4171/OWR/2014/22.

5. L. J. MORDELL, On the evaluation of some multiple series, J. London Math. Soc. 33 (1958), 368-371. MR 0100181

6. D. MuMFORD, Hirzebruch's proportionality theorem in the noncompact case, Invent. Math. 42 (1977), 239-272. MR 0471627

7. M. NIQUI, Exact arithmetic on the Stern-Brocot tree, J. Discrete Algorithms 5 (2007), no. 2, 356-379. MR 2317038

8. M. PASSARE, How to compute $\sum 1 / n^{2}$ by solving triangles, Amer. Math. Monthly 115 (2008), no. 8, 745-752. MR 2456096

9. C. L. SiEGEL, Symplectic geometry, Amer. J. Math. 65 (1943), 1-86. MR 0008094

10. D. ZAGIER, Values of zeta functions and their applications, First European Congress of Mathematics, Vol. II (Paris, 1992), Progr. Math., vol. 120, Birkhäuser, Basel, 1994, pp. 497-512. MR 1341859

\section{Photo Credits}

Photo of Jürg Kramer by Michael Ebner.

Photo of Anna-Maria von Pippich, courtesy of Anna-Maria von Pippich.
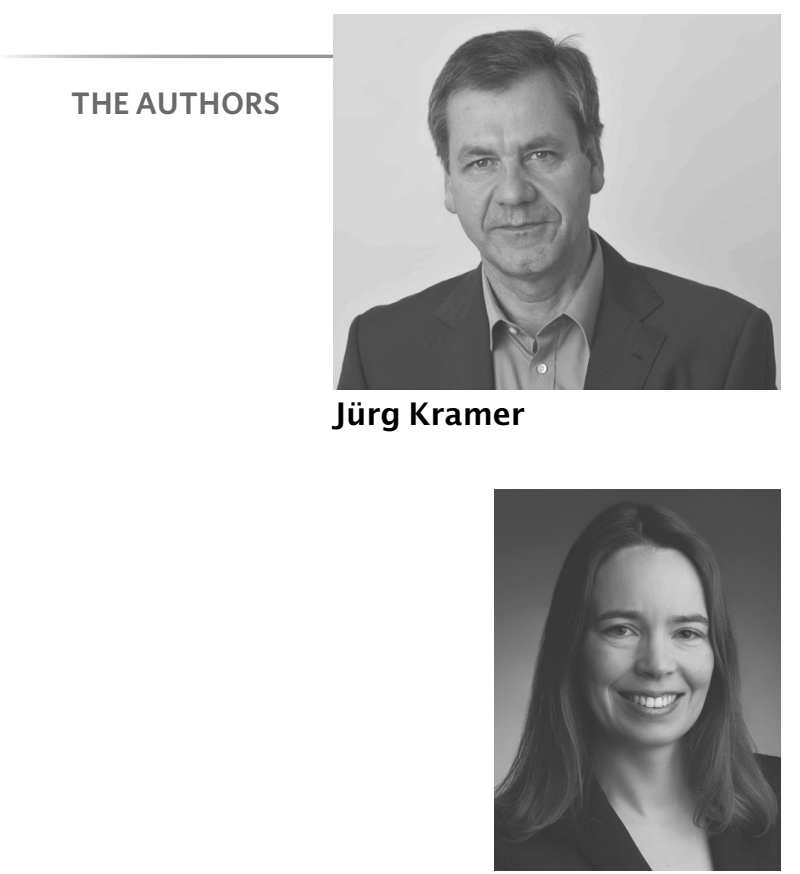

Anna-Maria von Pippich 